\title{
Pioneering carbon nanotube textile engineering \& fashion technology
}

\begin{abstract}
This paper describes innovative work in the manufacturing of carbon nanotube (CNT) fabric and tape and how these materials can be used in textiles and fashion apparel. The field of CNT fabric is new and this article discusses the potential commercial impact of CNT fabric, the manufacturing barriers that currently exist, and provides suggestions to push the field towards large scale commercialization. Areas under investigation for commercialization of CNT fabric, tape and yarn include flexible heaters, filter membranes, smart textiles, electrical machines, hybrid fabric, and biomedical wire.
\end{abstract}

Volume 5 Issue 2 - 2019

\author{
Megha Chitranshi, ${ }^{1,2}$ Devika Chauhan, ${ }^{1,2}$ \\ Ashley Kubley, ${ }^{3}$ Anuptha Pujari, ${ }^{1,2}$ Chenhao \\ $\mathrm{Xu},{ }^{1,2}$ Daniel Chen, ${ }^{1,2}$ Sumeet Chaudhary, ${ }^{1,2}$ \\ Guangfeng Hou, ${ }^{1,2}$ Gregory Bell, ${ }^{4}$ Brooke \\ Brandewie, ${ }^{3}$ Rutvik Kaneria, ${ }^{1,2}$ Ronald \\ Hudepohl,' Mark J Schulz',2 \\ 'College of Engineering and Applied Sciences, University of \\ Cincinnati, United States \\ ${ }^{2}$ Nanoworld Laboratories, University of Cincinnati, United \\ States \\ ${ }^{3}$ College of Design, Art, Architecture and Planning, University of \\ Cincinnati, United States \\ ${ }^{4} \mathrm{KCB}$ Capital Partners/Beckman Machine, United States
}

\begin{abstract}
Correspondence: Mark J Schulz, College of Engineering and Applied Sciences, Professor, Co-Director of Nanoworld Laboratories, University of Cincinnati, Cincinnati, Ohio 4522I, United States, Tel (5I3) 556-4I32,

Email schulzmk@ucmail.uc.edu
\end{abstract}

Received: March 17, 2019 | Published: March 26, 2019

\section{Introduction}

Carbon nanotubes (CNT) individually have extraordinary properties. However, macroscale fabric and tape made from CNTs have much reduced properties compared to individual CNTs. CNT in macroscale forms are also expensive to produce as the yield of the CNT synthesis process is low and assembling individual CNT into large size fabrics is complicated and expensive. Thus the two main barriers to putting CNT fabric into applications are to improve the material properties and to scale-up manufacturing yield to reduce cost. These aspects are discussed in this paper. Examples of CNT fabric and tape are shown in Figure 1. The tape can be twisted to form yarn which is stronger than tape.
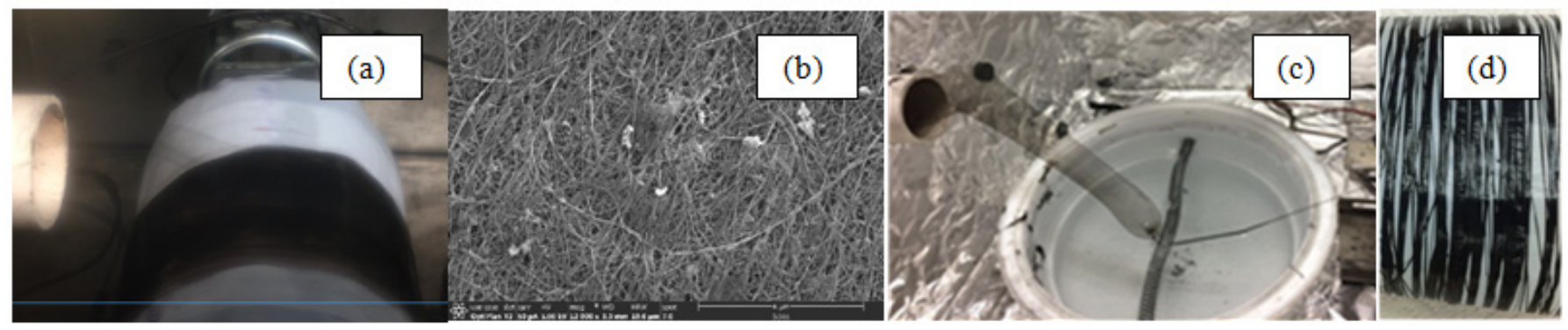

Figure I Manufacturing CNT material using the floating catalyst method: (a) CNT sock exiting the reactor and winding onto a Teflon sheet/drum; (b) to form nonwoven fabric; (c) CNT sock densified by drawing through water; (d) to form tape.

\section{Manufacturing barriers}

CNT sheet or yarn materials are manufactured ${ }^{1}$ within four main processes:

(i) Synthesis of powder CNT and using liquid dispersion and vacuum to form nonwoven sheet which is low cost with good electrical conductivity but modest strength;

(ii) Substrate growth of CNT forests and dry drawing to form sheet or drawing and twisting to form yarn which produces material with high purity and good strength and conductivity, but high cost; (iii) The floating catalyst method that produces a spider web or sock of CNT that can be wrapped to form sheet or pulled and twisted to form yarn which have good conductivity and strength but the process yield is low; and

(iv) Wet extrusion of CNT dispersions in super acid to form yarn which has high electrical conductivity and modest strength.

Carbon Hybrid Materials ${ }^{1}$ is a modified floating catalyst method wherein metal or ceramic particles are integrated within the CNT sheet during the synthesis process. ${ }^{2}$ The properties of the hybrid material are being evaluated. The floating catalyst method continuously produces CNT sock that is wound on a drum to form sheet or tape, or twisted 
to form yarn. This method has limitations including that the yield of the process is low. Larger size reactors are needed to reduce the cost of the material for commercialization. However, larger size ceramic tubes are too expensive and brittle. ${ }^{1}$ Thus a new break-out technology is needed to increase the throughput and reduce the cost of CNT sheet and yarn produced by the floating catalyst method, see Ch. 7 and $\mathrm{Ch}$. 23 in Nanotube Superfiber Materials. ${ }^{1}$

\section{CNT continuous synthesis}

The floating catalyst method is a continuous process that uses gas phase pyrolysis for CNT synthesis. In this process, feedstock is injected from one end of the ceramic tube furnace, and the synthesized CNT aerogel-like sock is collected at the other end in the form of sheet or tape. The feedstock is composed of a metal catalyst, ${ }^{3-5}$ hydrocarbon as a carbon source, and sulfur as a promoter. A carrier gas such as hydrogen, argon, helium, nitrogen or a mixture of two or more gases facilitate floating the atomized form of the feedstock and catalyst into the reactor. Also, the alignment of the ceramic tube inside the furnace can be either horizontal ${ }^{6}$ or vertical..$^{7}$ Along with these, the synthesis of CNTs uses different temperatures, ranging from $800^{\circ} \mathrm{C}^{8}$ to $1400^{\circ} \mathrm{C}$. The physical properties of the synthesized CNT material are highly dependent on the parameters mentioned.

\section{Material properties}

CNT Fabric is nonwoven material and thus is simpler to manufacture than woven or knitted fabrics. CNT yarn can also be woven to form stronger fabric but the cost of yarn is higher than sheet, and the weaving process further increases cost. CNT fabric and yarn have properties approximately as in Table 1. The properties vary depending on the exact manufacturing conditions. The properties are satisfactory for some applications, but increasing the properties is the goal in order to open up more applications and to compete with existing fibers and textiles.

Table I Approximate properties of CNT nonwoven sheet and CNT yarn

\begin{tabular}{ll}
\hline Property & Nanotube sheet and tapes \\
\hline Tensile Strength (GPa) & $0.4-\mathrm{I} .2$ \\
Elastic Modulus (GPa) & 100 \\
Strain to failure (\%) & Up to I5 \\
Electrical Resistivity (ohm cm) & $2 \times 10^{-4}$ \\
& $30-100$ in plane, 0.4-2 normal to the \\
Thermal Conductivity W/(mK) & plane, this extreme anisotropy in thermal \\
& conductivity is from 78:I to 50:I \\
Sheet Resistance (ohm/square) & 0.3 \\
Seebeck Coefficient (microV/K) & -60 n type, 70 p type \\
Density (g/cc) & $0.1-\mathrm{I} .2$, depends on solvent or other \\
densification \\
Burning Temperature in Air & High resistance to flame \\
Property & Nanotube Yarns \\
Tensile Strength (GPa) & $2-3$ (up to 4 in thin tapes) \\
Elastic Modulus (GPa) & 200 \\
Strain to failure (\%) & 4 \\
Electrical Resistivity (ohm cm) & IxI0-4 \\
Density (g/cc) & I.I \\
Thermal Conductivity W/(mK) & \\
\hline
\end{tabular}

\section{Commercialization}

CNT commercialization has found early success in aerospace and certain high value structural and electrical applications. ${ }^{3,4}$ Characteristics providing applications value include; strong, lightweight, flexible, electrically conductive, and chemical \& environmental resistance. Expansion into other applications requires further development and lower cost for CNT materials. Potential applications of CNT fabric and yarn include commercial products in the areas of technical textiles, fashion apparel, medicine, composite materials, electrical power conductors, electromagnetic shielding materials, carbon clothing, textile heater elements, EMI \& ESD protection, filtration, and others. While there are hundreds of companies' worldwide working with CNTs, the number of companies developing sheet and yarn is perhaps in the tens. A few companies in the USA are producing materials with different properties and at different price points. ${ }^{9-12}$ Other companies are using the fabric for applications such as electrical shielding of cables. ${ }^{13}$ Other applications in technical textiles and fashion apparel are listed in. ${ }^{1,14,15}$ CNT textiles hybridized with metal or ceramic particles are described in..$^{1,15-17}$ Carbon nanotube sheet is used in fiber reinforced composite structures, ${ }^{6,18}$ for delamination detection, ${ }^{19}$ and structural health monitoring. ${ }^{20}$ There is enormous potential of carbon nanotube materials in antenna and communication applications. ${ }^{21-23}$ Also, the integration of CNT energy storage and super capacitor applications $\mathrm{s}^{24-26}$ is recently on the rise. Moreover, these CNT communication devices, energy storage devices, and super capacitors can be integrated into textile and fashion apparel.

\section{Flexible heaters}

Due to the excellent electrical and thermal properties of CNT fabric, it can be integrated into textiles for heating applications. The role of CNT fabric is to provide conductive pathways in a garment which may be a composited material (containing layers of different fabrics). CNT fabric's porous nature can provide significant surface area of the material, and using its good mechanical properties and light weight, flexible CNT fabric heater material can be manufactured. CNT based heaters nominally use the same power as conventional heaters. But CNT heater fabric provides uniform heating without adding significant weight or size to garments. The heating temperature depends on the fabric thickness, length, and applied voltage. The resistivity of the fabric can be varied according to the application requirements to provide the needed power at the desired voltage level, per $\mathrm{P}=\mathrm{V}^{2} / \mathrm{R}$, where $\mathrm{P}=$ power dissipated as heat, $\mathrm{V}=$ applied voltage, and $\mathrm{R}=$ resistance of the fabric. Thus textiles may have built-in heaters.

\section{Filter membranes}

The problem of heavy metals in water has existed for a long time due to human activities and civilization. The metals are toxic to aquatic life and human beings. These pollutants do not degrade in a natural environment like some of the organic pollutants. ${ }^{27}$ Common heavy metals include $\mathrm{Cd}, \mathrm{Hg}, \mathrm{Ni}, \mathrm{Co}$, and $\mathrm{Pb}$. Among them, lead is the most common noxious metal ion. ${ }^{28}$ Industrial activities like battery manufacturing, acid metal plating and finishing, and tetraethyl lead manufacturing are the major sources ${ }^{29}$ of lead. The presence of lead harms the kidneys, liver, and nervous and reproductive systems. ${ }^{28}$ Therefore, it is necessary to minimize lead contamination to prevent potential threats to society. ${ }^{30} \mathrm{CNTs}$ are a relatively new absorbent which can remove heavy metals ${ }^{31}$ such as lead ions ${ }^{32}$ from water. The large surface area, high porosity, hollow structure, and strong interaction between contaminant molecules and CNTs are key features of CNT membranes for absorption and filtration applications. ${ }^{33,34}$ 
The adsorption properties of CNT membranes depend on the purity, porosity, surface area, and surface functional groups ${ }^{33}$ of the fabric. The implantation of surface functional groups can enhance the adsorption rate of heavy metals. ${ }^{33}$ Acid treatment has been widely used with acids like $\mathrm{HCl}, \mathrm{H}_{2} \mathrm{SO}_{4}, \mathrm{HNO}_{3}, \mathrm{KMnO}_{4}$, and $\mathrm{H}_{2} \mathrm{O}_{2} \cdot{ }^{35}$ Acid treatment introduces different functional groups onto the CNT membrane surface. These functional groups enhance CNT membranes which remove different contaminants. The adsorption capacity of acid treated CNT membranes is higher than that of raw CNT membranes because of the electrostatic interaction between the negative charge on the CNT's surface and the positively charged metal ions..$^{36,37}$ In addition, acid treatment also removes impurities from the raw CNTs. ${ }^{33}$ Furthermore, CNT membranes can be modified with other nanoparticles to improve the removal efficiency for heavy metals and natural organic matter (NOM). Air filtration is another application of CNT fabric [see Ch. 26 in Nanotube Superfiber Materials ${ }^{1}$ ]. Thus textiles may have filtering capabilities.

\section{CNT smart textile applications}

The development of composite textiles utilizing CNT has emerged at the forefront of functional and smart textiles research. The integration of CNT materials can extend the capabilities of functional textiles and create new applications for the consumer market. CNT can give textiles added performance value such as filtration capabilities for waterborne and airborne contaminants; wicking and heat spreading capabilities; electrical conductivity that can support integrated electronic components, and the possibility to harness and produce power. CNT impregnated and composited textiles exhibit extremely light weight with modest strength which gives textiles that include them an edge over existing materials. CNTs have the potential to harness and store energy to create flexible, lightweight power systems that do not need electricity to operate. The integration of CNTs in textiles can functionalize conventional fabrics without changing inherent fabric properties like softness and flexibility, making them discreet and easily integrated into fabric systems. These additive properties open the door to new possibilities for the design of consumer-driven smart products that can monitor body metrics, provide improved comfort and safety for the user and improved performance of the material. This added value gives CNT an appealing edge in the emergent Smart Textiles and Wearable Technology markets, which is growing rapidly. Materials that are sensory, reactive, self-powered and performance enhancing define a new class of functional textiles that are intelligent. CNT materials integrated into textiles can perform multiple enhancement functions at once. This creates potential for the development of multi-functional products in the apparel, home, occupational and specialized personal protective equipment markets.

\section{CNT textiles in electrical machines}

Copper is the most widely used conductor in electrical machines. However, despite having high conductivity at room temperature, the conductivity of copper decreases at higher temperatures thus making it unsuitable for high temperature operation. Moreover, copper has a high density of around $8.9 \mathrm{~g} / \mathrm{cc},{ }^{38}$ which means that products made of $\mathrm{Cu}$ carry a weight penalty. Also, when used at higher frequencies, $\mathrm{Cu}$ conductors suffer from the skin effect, a phenomenon in which high frequency $\mathrm{AC}$ current flows through the outer layer of the conductor effectively reducing the size of the conductor. A pioneering advance may be to have a machine that operates at high frequency, high voltage, and high temperature. It has been proposed to replace $\mathrm{Cu}$ conductors with CNT sheets, as the resistivity of CNT sheets is stable at higher temperatures and at the same time the structure of CNT inherently resembles multi-strand litz wires which are suitable for being used at high frequencies without suffering from the skin effect. CNT yarn also has a higher maximum current density than $\mathrm{Cu}$ wire. The downside of CNT wire is the high cost and it is less conductive than $\mathrm{Cu}$.

\section{CNT hybrid fabric}

The properties of CNT sheets produced using the floating catalyst method are satisfactory for some applications. In order to achieve better properties and enable greater applications, it is important to integrate CNT's with nanoparticles. There are different ways in which CNT's can be integrated with metal/alloy or ceramic nanoparticles but to do so it is important to understand the theory behind the integration of nanoparticles with CNT's. Wetting and solubility are two important factors to be taken into consideration. CNT's can be wet by low surface tension elements or compounds with a cut-off value of 200 millinewtons per meter $(\mathrm{mN} / \mathrm{m}){ }^{39}$ Systems of metals belonging to group VIII also wet CNT's due to the solubility of the solid phase in the liquid phase. ${ }^{40}$ Also, carbide forming metal melts can wet CNT's due to their formation of chemical compounds during the process of synthesis of CNT's. High surface tension liquids do not wet the CNT's, and these can be used to decorate the outer surface of the nanotubes. ${ }^{41}$ It is also important to consider the fact that contact angles and wetting properties are largely dependent on experimental conditions. Producing CNT hybrid fabric is a new area of research that will enable customizing the properties of textiles.

\section{CNT Yarn Biomedical Wire}

CNT yarn is being evaluated for use as biomedical wire. CNT wire has been used to cross the skin to connect to sensors in mice ${ }^{1}$, to transmit power from a battery to an implantable device in a pig, and as a scaffold for regenerating neurons. Other biomedical applications may include reinforcing bone, powering robots, pacemaker wire, and others. CNT wire may be used to transmit power or to communicate with devices implanted in the body. The wire can cross the skin and connect to Smart textile apparel to monitor and record information from the implanted device. The wire may also take signals from neurons in the body and connect to wearable electronics in smart textiles to diagnose or treat different medical conditions.

\section{Investing in new textile capability}

In the long term, investment in CNT fabric and yarn may be sensible commercially in various areas. Advances have been coming in CNT manufacturing from various research laboratories around the world. Synthesis of bundles of half-meter long CNT, carbon nanotube hybrid materials, control of chirality to produce mostly metallic CNT, high quality CNT with a high Raman G/D ratio, and high strength for thin sheets of CNT have been reported recently. Societal, intellectual, and equipment capital all play into an investment strategy, but overall the low yield and low properties of CNT fabric are the main barriers to be overcome. The current cost of CNT sheet is on the order of five thousand dollars per pound. The need for development of larger reactors, including simulation, experimentation, and prototyping, requires large investment possibly beyond the level of university research laboratories. The authors anticipate that the properties of CNT fabric and yarn will continue to improve through the efforts of the many research laboratories internationally, but the research findings must be integrated to take advantage of all the advances. In summary, convergence of research findings and efforts, and significant capital investment in scale-up of manufacturing may bring new CNT fabric into mainstream commercial textile use. 


\section{Acknowledgments}

This research was supported by the NSF ERC EEC-0812348 and UCTAC Seed Grant under ESP TECH 15-0160.

\section{Conflicts of interest}

Author declares there is no conflict of interest in publishing the article.

\section{References}

1. Mark J Schulz, Vesselin Shanov, John Yin, et al. Nanotube superfiber materials, science, manufacturing, commercialization. 2nd ed. Elsevier; 2019

2. Mark Schulz, Vianessa Ng, Guangfeng Hou. Methods of manufacturing carbon nanotube (CNT) hybrid sheet and yarn by gas phase assembly, and CNT-hybrid materials. PCT/US2018/019427; 2018.

3. Hou G, Chauhan D, Vianessa Ng, et al. Gas-phase pyrolysis synthesis of carbon nanotubes at high temperature. Mater Des. 2017;132:112-118.

4. Deepak FL, John NS, Govindaraj A, et al. Nature and electronic properties of Y-junctions in CNTs and N-doped CNTs obtained by the pyrolysis of organometallic precursors. Chem Phys Lett. 2005;411(4-6):468-473.

5. Sen R, Govindaraj A, Rao CNR. Carbon nanotubes by the metallocene route. Chem Phys Lett. 1997;267(3-4):276-280.

6. Chauhan D, Hou G, Vianessa Ng, et al. Multifunctional smart composites with integrated carbon nanotube yarn and sheet. Proceedings of SPIE. $2017 ; 10172$.

7. Conroy D, Moisala A, Cardoso S, et al. Carbon nanotube reactor: ferrocene decomposition, iron particle growth, nanotube aggregation, and scale-up. Chem Eng Sci. 2010;65(10):2965-2977.

8. Mohammadian N, Ghoreishi S, Hafeziyeh S, et al. Optimization of synthesis conditions of carbon nanotubes via ultrasonic-assisted floating catalyst deposition using response surface methodology. Nanomaterials. 2018;8(5):316

9. General Nano Company. Beyond performance.

10. Nanocomp Technologies.

11. American Boronite Company.

12. TE Connectivity Company. Carbon nanotube technology promise a revolution in cabling.

13. Lintec Company.

14. Sullivan J, Schulz M, Vemaganti K, et al. Carbon nanotube fabric cooling system for firefighters and first responders: modeling and simulation Journal of Fiber Bioengineering and Informatics. 2015;8:1-12.

15. Mark Schulz, Vianessa Ng, Guangfeng Hou, et al. Carbon nanotube hybrid material fabric, composite fabric, and personal protective apparel and equipment. 10738-620 PCT; 2018.

16. Chauhan $\mathrm{D}, \mathrm{Xu} \mathrm{C}$, Chen $\mathrm{D}$, et al. Introduction to carbon nanotube hybrid textiles. Journal of Textile Science \& Fashion Technology. 2019;1(5):17.

17. Chen D, Chauhan D, Hou G, et al. Introduction to carbon hybrid materials, nanotechnology materials and devices workshop. University of Cincinnati 2018.

18. Song Y, Chauhan D, Hou G, et al. Carbon nanotube sheet reinforced laminated composites. Proc of the American Society for Composites. 2016.

19. Abot JL, Song Yi, Vatsavaya MS, et al. Delamination detection with carbon nanotube thread in self-sensing composite materials. Compos Sci Technol. 2010;70(7):1113-1119.
20. Abot JL, Schulz MJ, Song Y, et al. Novel distributed strain sensing in polymeric materials. Smart Mater Struct. 2010;19(8):085007.

21. Keller SD, Zaghloul AI, Shanov V, et al. Radiation performance of polarization selective carbon nanotube sheet patch antennas. IEEE Trans Antennas Propag. 2014;62(1):48-55.

22. Amram Bengio E, Senic D, Taylor LW, et al. High-efficiency carbon nanotube thread antennas. Appl Phys Lett. 2017;111(16):163109.

23. Puchades I, Rossi JE, Cress CD, et al. Carbon nanotube thin-film antennas. ACS Appl Mater Interfaces. 2016;8(32):20986-20992.

24. Sun L, Wang X, Wang Y, et al. Roles of carbon nanotubes in novel energy storage devices. Carbon. 2017;122:462-474.

25. Jae-Hak Kim, LeeKH, Overzet LJ, et al. Energy storage devices using spinnable CNT sheets. 2011 IEEE Nanotechnology Materials and Devices Conference; 2011:45-49.

26. Wu G, Tan P, Wang D, et al. High-performance supercapacitors based on electrochemical-induced vertical-aligned carbon nanotubes and polyaniline nanocomposite electrodes. Sci Rep. 2017;7:43676.

27. Stafiej A, Pyrzynska K. Adsorption of heavy metal ions with carbon nanotubes. Seprartion and Purification Technology. 2007;58(1):49-52.

28. Zarghami S, Tofighy MA, Mohammadi T. Adsorption of zinc and lead ions from aqueous solutions using chitosan/polyvinyl alcohol membrane incorporated via acid-functionalized carbon nanotubes. Journal of Dispersion Science and Technology. 2015;36(12):1793-1798.

29. Chen YC, Lo SL, Luo J. Pb(II) adsorption capacity and behavior of titanate nanotubes made by microwave hydrothermal method. Colloids Surf A. 2010;361(1-3):126-131.

30. Li J, Chen $\mathrm{S}$, Sheng G, et al. Effect of surfactants on $\mathrm{Pb}$ (II) adsorption from aqueous solution using oxidized multiwall carbon nanotubes. Chem Eng J. 2011;166(2):551-559.

31. Savage N, Diallo MS. Nanomaterials for water purification. J Nanopart Res. 2005;7(4-5):331-342.

32. Li YH, Di Z, Ding J, et al. Adsorption thermodynamic, kinetic and desorption studies of $\mathrm{Pb} 2+$ on carbon nanotubes. Water Res. 2005;39(4):605-609.

33. Ihsanullah, Aamir A, Adnan MA, et al. Heavy metal removal from aqueous solution by advanced carbon nanotubes: critical review of adsorption applications. Separation and Purification Technology. 2016;157:141-161

34. Di ZC, Li YH, Luan ZK, et al. Adsorption of chromium (VI) ions from water by carbon nanotubes. Adsorpt Sci Technol. 2004;22(6):467-474.

35. Rao GP, Lu C, Su F. Sorption of divalent metal ions from aqueous solution by carbon nanotubes: a review. Sep Purif Technol. 2007;58(1):224-231.

36. Wang HJ, Zhou AL, Peng F, et al. Adsorption characteristic of acidified carbon nanotubes for heavy metal $\mathrm{Pb}$ (II) in aqueous solution. Mater Sci Eng A. 2007;466(1-2):201-206.

37. Ihsanullah, Khaldi FAA, Abusharkh B, et al. Adsorptive removal of cadmium(II) ions from liquid phase using acid modified carbon-based adsorbents. J Mol Liq. 2015;204:255-263.

38. Pyrhönen J, Montonen J, Lindh P, et al. Replacing copper with new carbon nanomaterials in electrical machine windings. International Review of Electrical Engineering (IREE). 2015;10(1).

39. Dujardin E, Ebbesen TW, Hiura H, et al. Capillarity and wetting of carbon nanotubes. Science. 1994:265(5180):1850-1852.

40. Mrityunjay S, Tatsuki O, Rajiv A, et al. Ceramic integration and joining technologies: from Macro to Nanoscale, 1st ed. Wiley-American Ceramic Society; 2012.

41. Ebbesen TW. Wetting, filling and decorating carbon nanotubes. Journal of Physics and Chemistry of Solids. 1996;57(6-8):951-955. 